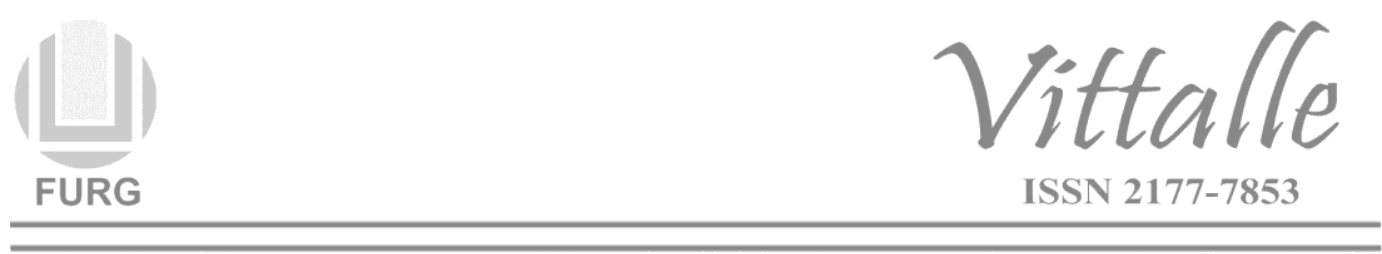

\title{
Metabolismo do triptofano em transtornos mentais: Um enfoque na esquizofrenia
}

\author{
Michelle S. Carvalho, ${ }^{\mathrm{a}, \mathrm{b}}$, Caroline Dal Mas ${ }^{\mathrm{a}, \mathrm{b}}$, Dayane F. S. Nunes ${ }^{\mathrm{b}}$, Camila Miyagui \\ Yonamine $e^{\mathrm{a}, \mathrm{b}}$, Mirian A. F. Hayashi ${ }^{\mathrm{a} \mathrm{b}^{*}}$ \\ ${ }^{a}$ Laboratório Interdisciplinar de Neurociências Clínicas (LiNC), Departamento de Psiquiatria \\ bDepartamento de Farmacologia, Universidade Federal de São Paulo (UNIFESP/EPM), São Paulo, São \\ Paulo, Brasil.
}

\section{Histórico do Artigo \\ Recebido \\ 09/01/2017 \\ Aceito em: 12/03/2017 \\ Palavras-chave \\ Triptofano \\ Quinurenina \\ Desordens psiquiátricas \\ Esquizofrenia}

Keywords

Tryptophan

Kynurenin

Psychiatric disorders

Schizophrenia

\section{RESUMO}

O triptofano (TRP) é um aminoácido essencial, encontrado no plasma sanguíneo, onde se encontra principalmente ligado à albumina, e com apenas uma pequena fração na forma livre. Ao atravessar a barreira hematoencefálica, seus metabólitos exercem diferentes ações no sistema nervoso central. Importantes metabólitos neuroativos resultantes do metabolismo do TRP (incluindo a serotonina, melatonina, 3-hidroxiquinurenina, ácido quinolínico e quinurênico, dentre outros) têm sido associados a doenças neurodegenerativas como a doença de Alzheimer e a de Huntington, e a doenças neuropsiquiátricas como o transtorno bipolar (TB) e a esquizofrenia (SCZ). Além disto, há também relatos sobre a sua relação com a depressão, doenças inflamatórias, doenças infecciosas e diversas alergias. O objetivo deste trabalho foi reunir informações recentes e atualizadas sobre o papel do TRP e seus metabólitos, enfatizando a relação desse aminoácido e seus metabólitos na patofisiologia da SCZ, com enfoque na via das quinureninas e sobretudo suas eventuais associações com as doenças mentais.

\section{Tryptophan Metabolism in mental disorders: A Focus on Schizophrenia}

\section{ABSTRACT}

Tryptophan (TRP) is an essential amino acid, mainly found bound to albumin in the blood plasma, in which only a small fraction is found at its free form. The TRP metabolites cross the blood brain barrier to perform different actions in the central nervous system. Important neuroactive metabolites derived from the TRP metabolism (including serotonin, melatonin, 3hydroxy kynurenine, quinolinic and quinurenic acid, among others) have been associated with neurodegenerative diseases as the Alzheimer's and Huntington's diseases, and with neuropsychiatric diseases as bipolar disorder (BD) and schizophrenia (SCZ). In addition, there are evidences showing that TRP and its metabolites are related to depression, inflammatory diseases, infectious diseases and with several allergies. The aim of this work was to summarize updated information on the role of TRP and its metabolites, highlighting the influence of this amino acid and its metabolites in the pathophysiology of SCZ, with focus on the kynurenin pathway and mainly in their association with mental diseases.

\section{Introdução}

A esquizofrenia (SCZ) é um transtorno mental grave, que afeta cerca de 0,5 a $1 \%$ da população mundial e com grande prejuízo social e econômico $(1,2)$. O diagnóstico da SCZ é baseado principalmente em relatos do paciente/cuidadores e histórico familiar, somado ainda à avaliação clínica, que consiste em entrevistas aplicadas por psiquiatras treinados, e que, portanto, são subjetivas por sua própria natureza (3). Alucinações, delírios, déficits cognitivos são alguns dos sintomas observados nesta patologia, considerada clinicamente complexa e heterogênea (4). A severidade dos sintomas em pacientes portadores de SCZ, em geral, são mensurados utilizando as Escalas das Síndromes Positiva e Negativa (PANSS) $(5,6)$, além de outros sistemas de diagnóstico $(7,8)$. Entretanto, os mecanismos moleculares envolvidos na fisiopatologia e no início da doença ainda não são bem conhecidos.

\footnotetext{
*Autor correspondente: mhayashi.unifesp@gmail.com (M. A. F. Hayashi)
} 
O triptofano (TRP) é um aminoácido essencial, ou seja, não é sintetizado pelo organismo, e, portanto, precisa ser obtido na dieta, e está presente na grande maioria dos alimentos. Cerca de $90 \%$ do TRP ingerido encontra-se ligado à albumina do plasma, e apenas $10 \%$ é encontrado na forma livre na circulação sanguínea (9)._Os ácidos graxos não esterificados são fundamentais para o equilíbrio entre a proporção do TRP na forma livre ou ligado à albumina, uma vez que competem com este aminoácido pela ligação com a albumina plasmática, aumentando consequentemente o nível de TRP livre no plasma $(10,11)$.

O TRP é substrato para a produção de diversas moléculas neuroativas com atividades biológicas, como a serotonina (5-HT), a melatonina, a 3-hidroxiquinurenina (3-HK), o ácido quinolínico (AQ), ácido quinurênico (QUINA), dentre outros (12,13) (Figura 1). As duas principais vias de metabolização do TRP conhecidas são a da 5-HT e da quinurenina (QUIN). Em torno de $95 \%$ do TRP obtido da dieta é metabolizado pela via da QUIN, e cerca de apenas $1 \%$ do TRP é convertido em 5-HT no sistema nervoso central (SNC), enquanto o restante é direcionado à síntese de proteínas e de melanina $(14,15)$.

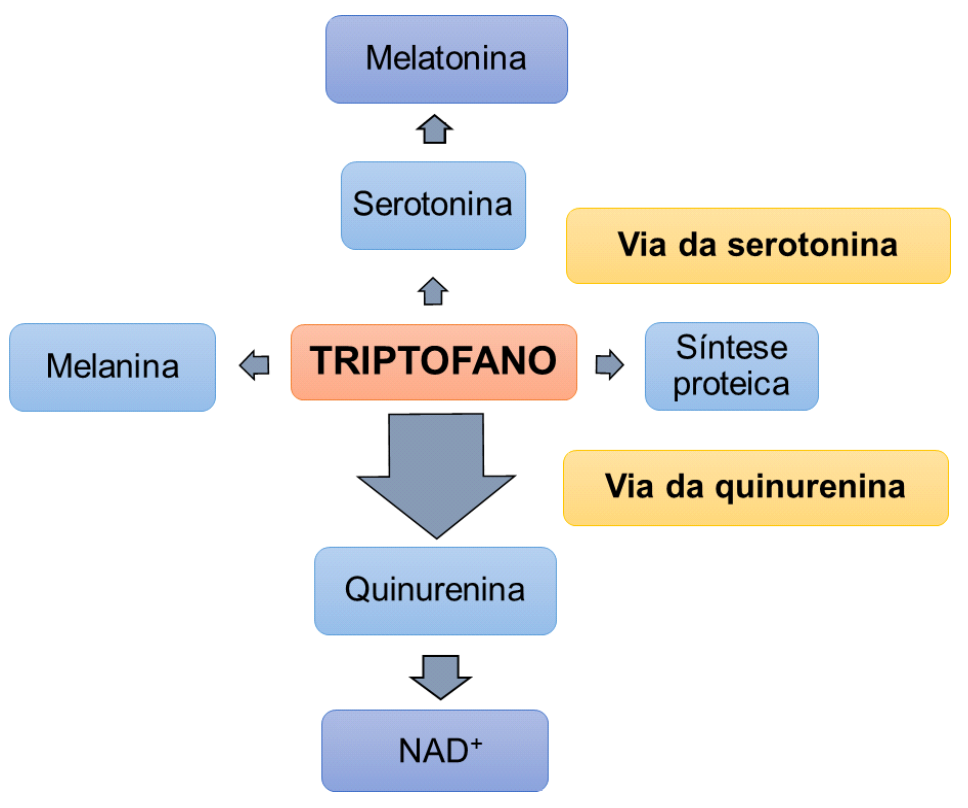

Figura 1. Vias de metabolização do triptofano. Na via da serotonina, o triptofano é convertido em serotonina e melatonina. Na via da quinurenina, o triptofano gera a quinurenina e derivados como a $\mathrm{NAD}^{+}$(nicotinamida adenina dinucleotídeo). Parte do triptofano ingerido na dieta serve ainda como fonte para a síntese de melanina e também como substrato para a síntese proteica.

Embora os estudos sobre o metabólitos do TRP sejam, em geral, centralizados na via da 5-HT, devido à sua importância como neurotransmissor (14), as vias de metabolização do TRP já foram associadas a doenças neurodegenerativas como a doença de Alzheimer (15) e a de Huntington $(16,17)$. Alterações nos níveis do TRP e em suas vias, já foram descritas em doenças infecciosas como a imunodeficiência viral humana (HIV) (18), em alergias (19), na depressão (20), em doenças auto-imunes como o lúpus (21), e em inflamações diversas $(22,23)$. Nos últimos anos, a via das QUINs e do metabolismo do TRP vêm ganhado destaque como um importante regulador da produção de agentes neuroprotetores (como o ácido quinurênico, o ácido picolínico e o cofator $\mathrm{NAD}^{+}$) e neurotóxicos (como o $\mathrm{AQ}$ e a 3-HK). $\mathrm{O}$ equilíbrio entre a produção destes metabólitos é controlado pela indolamina-2,3-dioxigenase (IDO) e sinais moleculares tais como interferon alfa (IFN- $\alpha$ ) responsáveis por ativar o metabolismo 
das QUINs em oposição as vias alternativas para a produção de 5-HT e melatonina. O AQ e QUINA têm sido relacionados à fisiopatologia de doenças mentais como a SCZ (24) e o transtorno bipolar (TB) (25).

\section{Metodologia}

Foi realizada revisão bibliográfica em bancos de bases de dados como PubMed, e Google Scholar. As buscas foram realizadas utilizando palavras chave como "esquizofrenia", "triptofano" e "quinurenina", no período de agosto a novembro de 2016. Em cada busca, foram obtidos aproximadamente 125.109, 56.562 e 4.392 resultados, respectivamente. A busca com as três palavras chave ao mesmo tempo, e com a busca concentrada em publicações de 2012 a 2017, permitiu a triagem de 1.730 artigos no Google Academics e 68 artigos no PubMed, sendo estes últimos todos também presentes na busca no Google Scholar. A triagem foi feita através da leitura prévia dos resumos, a fim de buscar artigos pertinentes para a composição deste trabalho. Foram selecionados preferencialmente trabalhos publicados a partir de 2012. Entretanto, isso não impediu a citação de trabalhos de grande relevância, com datas anteriores a 2012.

\section{Resultados e discussão}

\section{Via da Serotonina}

Conforme mencionado, o triptofano (TRP) é substrato para a síntese de 5hidroxitriptamina, também conhecido como serotonina (5-HT), no sistema nervoso central (SNC) (Figura 2). A disponibilidade do TRP pode exercer efeitos limitantes na taxa de síntese de 5-HT $(12,13)$. O TRP é hidrolisado pela ação da enzima triptofano hidroxilase (TPH) em 5-hidroxitriptofano (5-HTP), que é rapidamente metabolizado a 5-HT (26). A 5-HT é armazenada em vesículas sinápticas, nos neurônios serotoninérgicos, até a sua utilização e/ou a sua metabolização em ácido 5-hidroxiindoleacético (5-HIAA) (Figura 2).

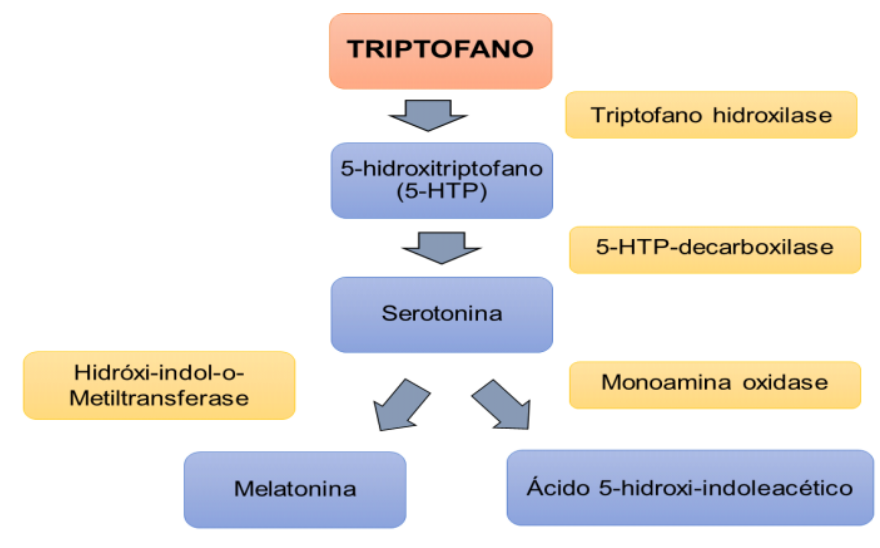

Figura 2. Metabolismo do triptofano (TRP). O TRP é a molécula precursora para formação do neurotransmissor serotonina e também do hormônio melatonina.

Eventualmente, o TRP ainda pode ser convertido em melatonina, que é um hormônio sintetizado pela glândula pineal, e é um hormônio relacionado à regulação do sono em 
humanos (27). É importante mencionar ainda que, embora sabidamente encontrada em concentrações menores em pacientes SCZ, a importância da melatonina para alguns sintomas específicos da SCZ e na inibição de alguns efeitos colaterais de antipsicóticos é ainda subestimada (28). Interessantemente, a via da melatonina é também reconhecida como fator de susceptibilidade genética para o TB, contribuindo para o risco aumentado de complicações cardiovasculares e diminuição da expectativa de vida (29), também frequentemente relatados na SCZ (30).

Quando há deficiência de niacina (vitamina B3) no organismo, o TRP pode ainda ser utilizado para síntese do cofator essencial nicotinamida adenina dinucleotídeo $\left(\mathrm{NAD}^{+}\right)$, que é um importante transportador de elétrons, e fundamental na produção de energia para a célula (31) (Figura 1).

\section{Via das quinureninas (QUIN)}

A via das QUIN é a principal rota de metabolização do TRP (Figura 3), sendo responsável pela metabolização de mais de $95 \%$ do TRP no organismo humano e a ação catabólica de algumas enzimas importantes desta via são dependentes da presença de vitaminas B2 e B6, que agem como cofatores (32).

Estudos recentes, conduzidos por Chiappelli e colaboradores (32), sugerem que os níveis de TRP totais são menores no plasma de pacientes portadores de SCZ, enquanto que a razão TRP/QUIN é maior, nesses pacientes, se comparados aos controles saudáveis, em jejum.

A metabolização do TRP pela via da QUIN pode ainda contribuir para uma menor integridade estrutural e funcional da substância branca observada em pacientes portadores de SCZ, sugerindo uma possível correlação entre esta alteração estrutural e os menores níveis de TRP e da maior razão TRP/QUIN em plasma de pacientes SCZ (32).

A via da QUIN está alterada em grande número de doenças neuropsiquiátricas, e a produção de mediadores inflamatórios, como as citocinas e quimiocinas, é capaz de aumentar a atividade da enzima indoleamina-2,3-dioxigenase (IDO) (20,24,29), que é fundamental para o metabolismo do TRP e a sua conversão em QUIN nos diferentes tecidos (12). 


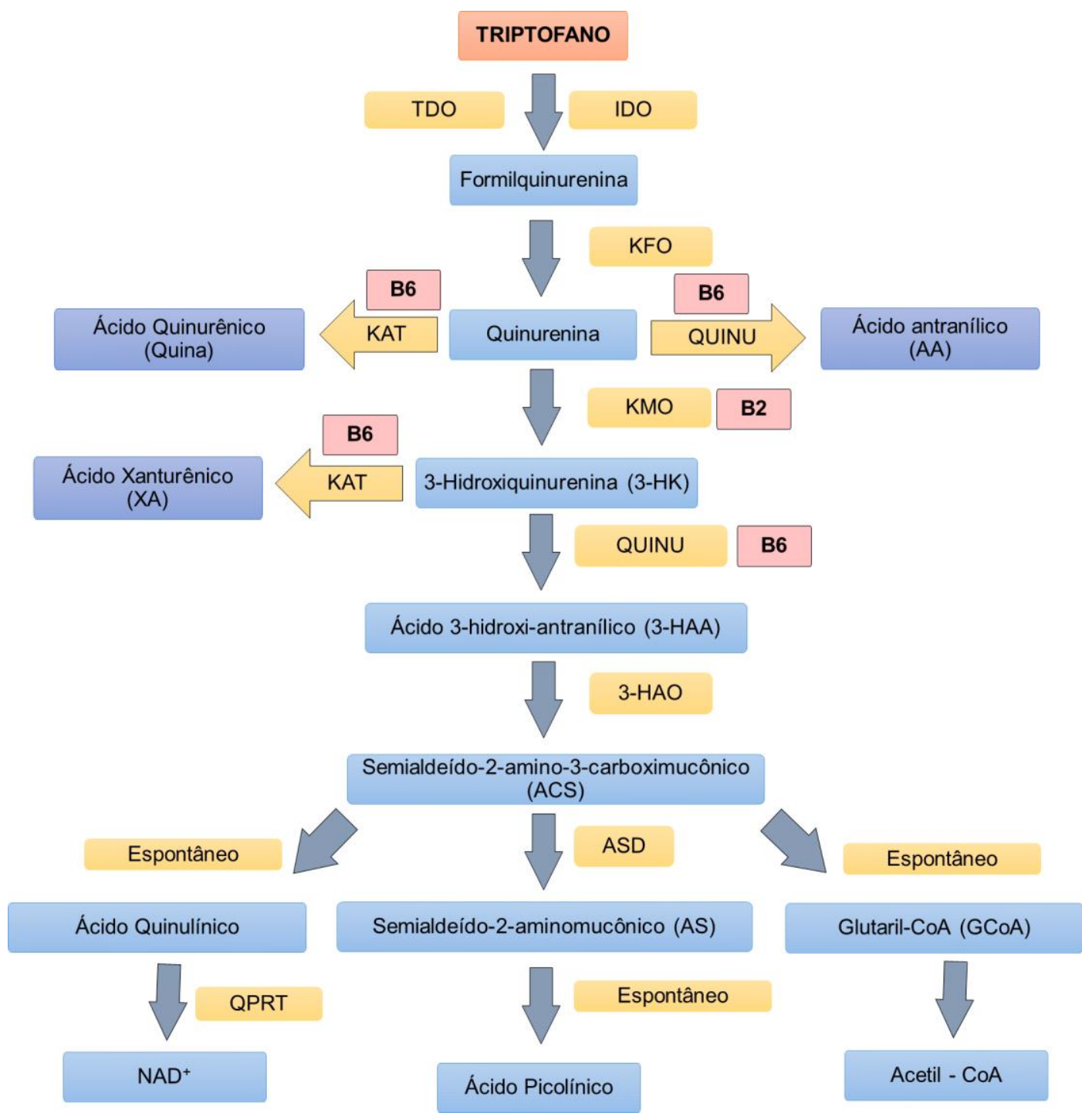

Figura 3. Metabolismo do triptofano (TRP): na via da quinurenina (QUIN), dando origem a diferentes metabólitos secundários. Reações mediadas pela ação das enzimas: TDO - triptofano-2,3-dioxigenase; IDO - indoleamina-2,3-dioxigenase; KAT - quinurenina transferase; KFO - quinurenina formam idase; KMO - quinurenina-3-monoxigenase; QUINU - quinureninase; 3-HAO - 3-hidroxiantranílico-3,4dioxigenase; ACS - semi-aldeído2-aminocarboximucônico; AS - semialdeído-2-aminomucônico; NAD ${ }^{+}$nicotinamida adenina dinucleotídeo; QPRT - quinolinato fosforibosil transferase, e pelos cofatores: B6 piridoxina e B2 - riboflavina.

Além de ativar a via da QUIN, as citocinas depletam o TRP, que é o precursor primário da 5-HT, e geram metabólitos neuroativos que podem influenciar significativamente na regulação da via da dopamina (DA) e do glutamato (GLU). A catálise do TRP em QUIN pode estar associada a sintomas e déficits cognitivos observados em transtornos mentais como a depressão e a SCZ ou TB (20,33-36).

O catabolismo da QUIN se inicia com a formação da n-formil-QUIN, pela ação das enzimas TRP 2,3-dioxigenase (TDO) ou pela IDO (37). A conversão do TRP em QUIN ocorre no fígado por ação da enzima TDO, enquanto que nos demais tecidos, como no cérebro, pulmão, coração, rim e no intestino, a principal enzima responsável por essa reação seja a IDO (37). Grant e colaboradores (38) demonstraram ainda que a diminuição da atividade da TDO é dependente do aumento na atividade da IDO e viceversa. 
A ausência da expressão da enzima quinurenina 3-hidroxilase em astrócitos (células da neuroglia), direciona à QUIN para a formação de ácido quinurênico (QUINA) (38). A QUIN pode ainda resultar em ácido antranílico (AA) pela ação da quinureninase (QUINU), e em 3-hidroxiquinurenina (3-HAA) pela quinurenina-3-monoxigenase (KMO). O QUINA produzido pelos astrócitos é captado pelas células da micróglia, resultando na síntese do ácido quinolínico (AQ) $(38,39)$.

A formação do AQ no cérebro ocorre principalmente nas células da micróglia (que são as menores células da neuroglia), e por inibição da transmissão sináptica, o AQ pode induzir a morte de células neuronais, degeneração neuronal e proliferação da glia (40). A administração intra-estriatal do AQ pode promover neurodegeneração, proliferação glial e disfunção cognitiva em animais, semelhante aos efeitos observados em portadores de SCZ $(39,40,41)$.

A via da QUIN está relacionada à fisiopatologia de diversas doenças mentais, devido à sua habilidade em promover tolerância imunológica ou neurotoxicidade. As células endoteliais e os pericitos presentes na barreira hematoencefálica (BHE) sintetizam constantemente a QUIN, e o seu excesso pode ser metabolizado por macrófagos e pela micróglia para a síntese do AQ, que é um agente neurotóxico (42).

\section{Efeitos neurotóxicos e neuroprotetores dos metabólitos do triptofano}

A QUIN periférica atravessa a BHE e, portanto, acessa facilmente o SNC. Uma vez no cérebro, a QUIN é absorvida pelas células da glia, onde sua metabolização é iniciada. Ao longo da via da QUIN, os metabólitos como ácido 3-hidroxiquinurenina (3-HK) e AQ são reconhecidos por seus efeitos neurotóxicos, podendo promover neurodegeneração $(13,43,44)$.

A neurotoxicidade da 3 -HK e do AQ foi demonstrada tanto em modelos in vivo $(43,45)$ quanto in vitro $(44,46)$. O aumento nos níveis de AQ e 3-HK no fluido cerebrospinal humano foi correlacionado com disfunções cognitivas e motoras, nas doenças de Huntington e Parkinson, e em doenças psiquiátricas como ansiedade, depressão e SCZ $(46,47)$. Acredita-se que a neurotoxicidade da 3-HK ocorra devido à sua capacidade de induzir estresse oxidativo e apoptose neuronal (46). A interação da 3HK com xantina oxidase (XO), produzindo espécies reativas de oxigênio (ROS), como o radical superóxido $\left(\mathrm{O}^{2-}\right)$ e peróxido de hidrogênio $\left(\mathrm{H}_{2} \mathrm{O}_{2}\right)$, induz a clivagem do DNA nuclear, promovendo a apoptose (46).

O excesso de ROS pode comprometer a ativação de receptores de membrana acoplados à proteína-G, além de favorecer a peroxidação lipídica e reduzir a função de receptores catecolaminérgicos e serotoninérgicos (41). O AQ é agonista do receptor Nmetil-D-aspartato (NMDA) e a estimulação desses receptores glutamatérgicos aumenta o influxo de cálcio nos neurônios, contribuindo para a geração de ROS e radicais livres; estimulando ainda a peroxidação lipídica da membrana, e comprometendo, assim, a sua fluidez e permeabilidade, o que pode, eventualmente, conduzir ao dano neuronal $(48,49)$ (Figura 4).

O QUINA também é um produto intermediário da metabolização da QUIN, e, ao contrário do AQ, que é um agonista, o QUINA age como antagonista dos receptores NMDA e, portanto, exerce ação neuroprotetora na SCZ (28). O QUINA é sintetizado e liberado por astrócitos e também exerce ação como antagonista em receptores $\alpha 7$ nicotínico de acetilcolina $(\alpha 7 \mathrm{nAChR})(47,50)$.

Foi demonstrado em roedores que o aumento ou a redução nos níveis de QUINA está associado à diminuição de DA extracelular e ao aumento na liberação de GLU e, consequentemente, a desregulação do QUINA endógeno contribui para a fisiopatologia 
de vários distúrbios, como a doença de Alzheimer, a doença de Parkinson, a SCZ, e a doença de Huntington $(51,52)$.

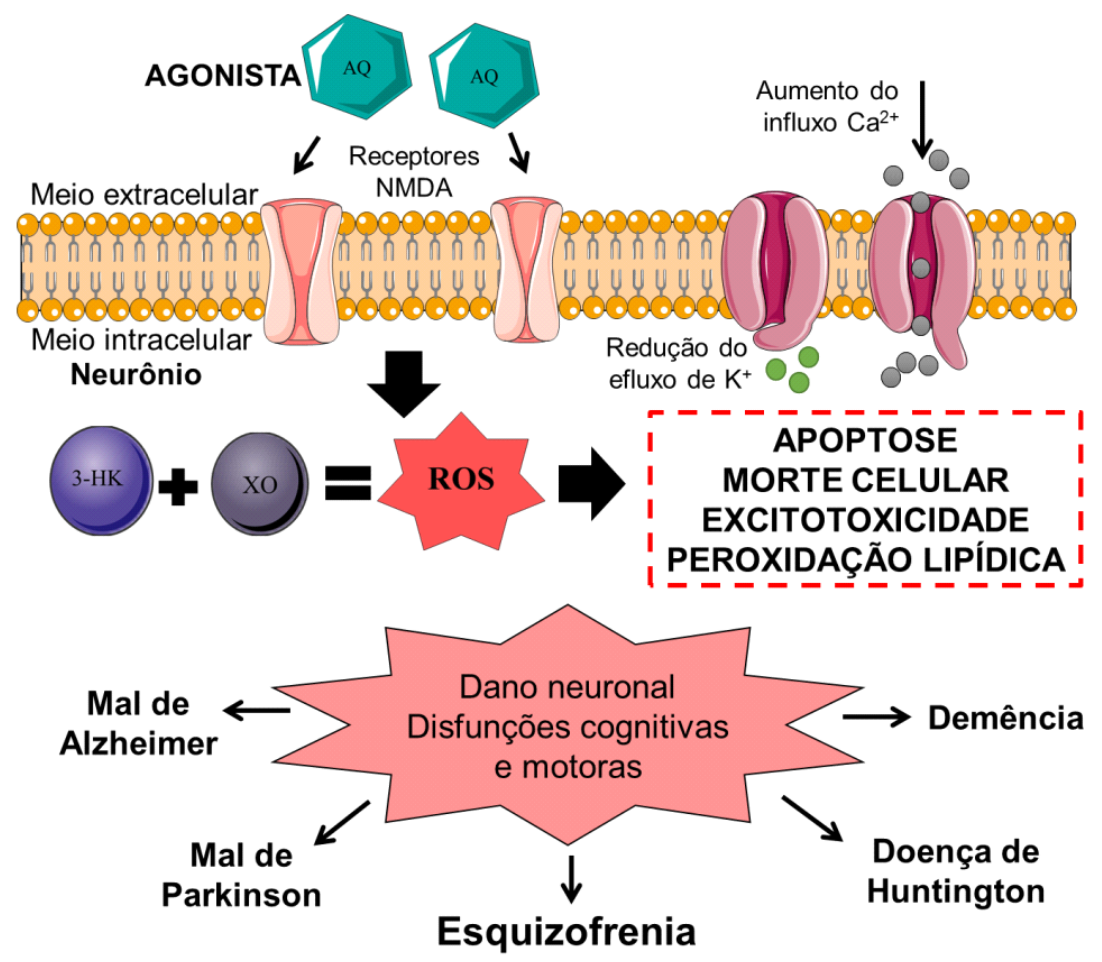

Figura 4. Efeito das espécies reativas de oxigênio em neurônios glutamatérgicos: A metabolização da quinurenina (QUIN) gera metabólitos neurotóxicos como o ácido 3- hidroxiquinurenina e ácido quinolínico (3-HK e o AQ) que podem promover neurodegeneração. A neurotoxicidade da 3-HK deve-se à indução do estresse oxidativo e apoptose neuronal. A interação da 3-HK com xantina oxidase (XO) produz espécies reativas de oxigênio (ROS), como o radical superóxido $\left(\mathrm{O}_{2}^{-}\right)$, o radical hidroxila $(\mathrm{HO} 2)$ e radicais livres, capazes de clivar o DNA nuclear e promover apoptose, com consequente dano neuronal e promoção de disfunções cognitivas e motoras.

Wang e colaboradores (23) identificaram o QUINA como um ligante endógeno do GPR35 (receptor 35 acoplado à proteína G). A ativação de GPR35 inibe a liberação do fator de necrose tumoral alfa (TNF- $\alpha$ ) por macrófagos, sob condições inflamatórias e destaca a importância do QUINA na regulação das funções imunes, sugerindo o possível efeito anti-inflamatório do QUINA (23). Por sua vez, Ohshiro e colaboradores (53) mostraram que a ativação do GPR35 diminui o $\mathrm{Ca}^{2+}$ intracelular, provavelmente pela inibição do influxo desse íon (53), corroborando com a hipótese de que o QUINA exerça efeito sobre a liberação de mediadores inflamatórios e aminoácidos excitatórios pelas células gliais.

Evidências sugerem que a QUIN possa ser considerada neuroprotetora devido ao seu potencial como antioxidante endógeno, por ser capaz de doar elétrons e proteger macromoléculas contra os efeitos oxidativos, como demonstrado tanto in vivo quanto in vitro $(54,55)$. Entretanto, essas propriedades podem ser independentes da formação de QUINA $(54,55)$ (Figura 5). As citocinas (IL-4 e IL-10) reduzem a atividade da IDO, levando à redução do catabolismo do TRP, que pode não comprometer a produção de 5HT (55). Entretanto, o TNF- $\alpha$, interferon alfa (IFN- $\alpha$ ) e TNF- $\alpha$ estimulam a atividade da IDO, aumentando o metabolismo de TRP, comprometendo, assim, a síntese de 5-HT, por favorecer a produção de metabólitos neurotóxicos como 3-HK e AQ (55). 


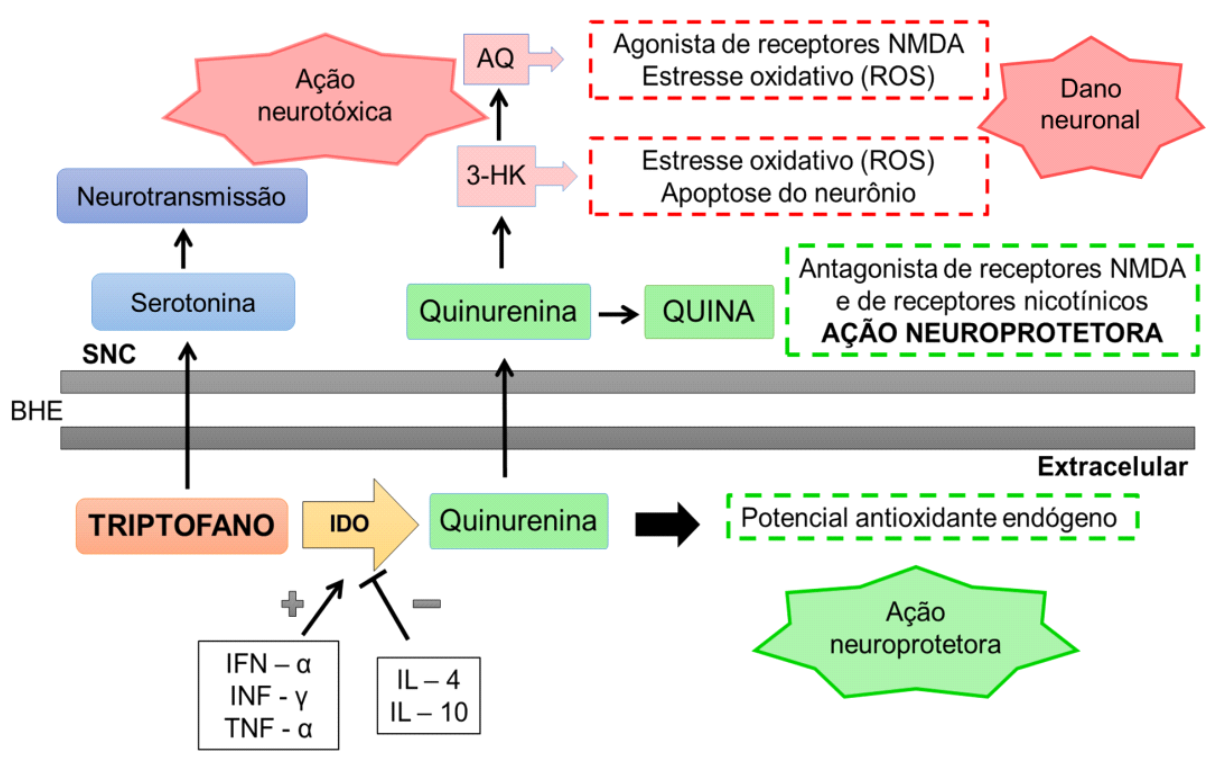

Figura 5. Efeito neurotóxico e neuroprotetor dos metabólitos do triptofano (TRP): A quinurenina (QUIN) periférica bem como o TPR atravessam a barreira hematoencefálica e atingem o sistema nervoso central (SNC), onde serão metabolizados. As interleucinas 4 e 10 (IL-4 e IL-10) reduzem a atividade da IDO, consequentemente reduzindo o catabolismo do TRP, sem comprometer a produção de 5-HT. Entretanto, o IFN- $\alpha$ e o TNF- $\alpha$ estimulam a atividade da IDO, aumentando o metabolismo de TRP, comprometendo, assim, a síntese de 5-HT e favorecendo a via da QUIN e a produção de metabólitos neurotóxicos como o ácido 3- hidroxiquinurenina e o ácido quinolínico (3-HK e AQ). O ácido quinurênico (QUINA) age como antagonista dos receptores NMDA (N-metil-D-aspartato) e dos receptores $\alpha 7$-nicotínico de acetilcolina $(\alpha 7 \mathrm{nAChR})$, exercendo ação neuroprotetora.

\section{Metabolismo do triptofano (TRP) e a esquizofrenia (SCZ)}

A SCZ caracteriza-se por sinais e sintomas complexos. Os sintomas positivos incluem alucinações (geralmente na forma de vozes), delírios (frequentemente paranoicos), comportamento bizarro e distorções perceptuais. Os sintomas negativos são evidenciados pela diminuição ou perda das funções, incluindo alogia, avolição, apatia, anedonia, emoções e reclusão social. Os portadores de SCZ têm, ainda, comprometimento significativo das funções cognitivas, incluindo déficits de memória e atenção, falta de discernimento/julgamento das funções executadas $(56-59,24)$.

A mais antiga hipótese que tenta explicar a fisiopatologia da SCZ é a via dopaminégica. A observação de que fármacos neurolépticos inibem os receptores de dopamina D2 e que antagonistas, como a anfetamina, favorecem o aumento de dopamina em portadores de SCZ, explica os sintomas positivos da doença. Posteriormente, a hipótese clássica foi reformulada quando Weinberger, em 1987 (59), sugeriu que um déficit na transmissão de DA nos receptores D1, no córtex pré-frontal, poderia esclarecer os sintomas negativos e alterações cognitivas dessa doença (59).

A redução plasmática do TRP tem sido atribuída ao aumento na conversão desse aminoácido em metabólitos derivados da QUIN $(60,61)$. Entretanto, a compreensão de como o metabolismo do TRP contribui para um maior entendimento da fisiopatologia de doenças como a SCZ ainda é bastante limitada.

Entretanto, já se sabe que a degradação do TRP e a ativação da via da QUIN gera vários compostos neuroativos como o QUINA, que é um antagonista do receptor NMDA e do receptor colinérgico alfa-7-nicotínico. O efeito do QUINA na SCZ pode ser explicado pela sua ação bloqueadora sobre os receptores de glutamato, que se encontram em níveis elevados no portador de SCZ. O QUINA compromete a 
neurotransmissão glutamatérgica e dopaminérgica, e a sua elevação no cérebro está relacionada a sintomas psicóticos e deficiências cognitivas (57-59).

A redução dos níveis plasmáticos de TRP em indivíduos $\mathrm{SCZ}$ resistentes ao tratamento com antipsicóticos foi relatada por Lee e colaboradores (62), o qual ponderou que o efeito fosse possivelmente determinado pelo aumento do estresse oxidativo frequentemente observado na SCZ (62). Por outro lado, há, ainda, aumento da concentração de QUIN e QUINA no tecido cerebral e no fluido cerebrospinal em pacientes com SCZ, comparados a controles saudáveis (63). Resultados contraditórios de concentrações maiores e menores de QUINA no plasma de portadores de SCZ são relatados (64-66), mas a relação entre as concentrações desses metabólitos do TRP no plasma, fluido cerebrospinal e outros tecidos (incluindo cérebro) não foram adequadamente estudados, ainda mais considerando que o QUINA não é capaz de passar a BHE (67). Embora os níveis séricos de outros metabólitos como o 3-HK diminuam após a terapia com antipsicóticos $(66,68)$ e do fato desse metabólito ter sido proposto como potencial biomarcador para predizer a severidade dos sintomas clínicos em pacientes em primeiro episódio de SCZ que não tenham feito uso de neurolépticos (69), não encontramos, em nossas buscas, relatos de alterações dos níveis de TRP nas suas vias metabólicas relacionadas com a etiologia da SCZ.

A ativação da IDO e a regulação do metabolismo da via da QUINA têm sido atribuídas à ação moduladora de células-T específicas, capazes de gerar citocinas próinflamatórias que podem contribuir diretamente para a desmielinização dos neurônios hipotalâmicos (56).

O aumento da QUIN no início do desenvolvimento pode promover diminuição da atividade glutamatérgica, resultando em déficits cognitivos em animais, que podem ser correlacionados às alterações cognitivas observadas em pacientes portadores de SCZ $(51,52)$.

Embora os dados deste estudo não sejam suficientes para identificar os mecanismos envolvidos, é possível sugerir que o baixo nível de TRP esteja relacionado à diminuição da oferta e ao aumento do catabolismo desse aminoácido, podendo promover, assim, maior vulnerabilidade para lesões da substância branca em pacientes, por ativação de vias de neuroinflamação, neurovasculares e neuroendócrinas (33).

\section{Considerações finais}

Os mecanismos envolvidos na via do TRP e da QUIN e a sua relação com a SCZ ainda são pouco conhecidos. Estudos mais específicos são fundamentais para conhecermos o seu real papel na fisiopatologia desse distúrbio mental. Como pode ser observado aqui, investigações para o melhor conhecimento dessa via têm potencial para contribuir com o entendimento da fisiopatologia da SCZ, contribuindo, ainda, para o diagnóstico e a geração de métodos mais eficazes de tratamento desse e de outros transtornos mentais.

Agradecimentos: Agradecemos o apoio financeiro das agências de fomento FAPESP, CNPq e CAPES.

Conflito de interesses: Os autores relatam nenhum conflito de interesses. Os autores são os únicos responsáveis pelo conteúdo e pela redação do artigo.

\section{Referências}

1. Chong HY, Teoh SL, Wu DB, Kotirum S, Chiou CF, Chaiyakunapruk N. Global economic burden of schizophrenia: a systematic review. Neuropsychiatr Dis Treat. 2016; 12:357-73. 
2. Whiteford HA, Degenhardt L, Rehm J, Baxter AJ, Ferrari AJ, Erskine HE, et al. Global burden of disease attributable to mental and substance use disorders: findings from the Global Burden of Disease Study 2010. Lancet. 2013; 382(9904):1575-86. Review.

3. Biedermann F, Fleischhacker WW. Psychotic disorders in DSM-5 and ICD-11. CNS Spectr. 2016; 21(4):349-54.

4. Galderisi S, Rossi A, Rocca P, Bertolino A, Mucci A, Bucci P, et al. The influence of illness-related variables, personal resources and context-related factors on real-life functioning of people with schizophrenia. World Psychiatry. 2014; 13: 275-287.

5. Kay SR, Fiszbein A, Opler LA. The positive and negative syndrome scale (PANSS) for schizophrenia. Schizophr. Bull. 1987; 13:261-276

6. Higuchi CH, Ortiz B, Berberian AA, Noto C, Cordeiro Q, Belangero SI, et al. Factor structure of the Positive and Negative Syndrome Scale (PANSS) in Brazil: convergent validation of the Brazilian version. Rev. Bras. Psiquiatr. São Paulo, 2014; 36 (4): 336-339.

7. Bressan RA, Chaves AC, Shirakawa I, de Mari J. Validity study of the Brazilian version of the Calgary depression scale for schizophrenia. Schizophr. Res. 1998; 32, 41-49.

8. Lima MS, Soares BG, Paoliello G, Machado Vieira R, Martins CM, Mota Neto JI, et al. The Portuguese version of the clinical global impression-schizophrenia scale: validation study. Rev. Bras. Psiquiatr. 2007; 29: 246-249.

9. Madras BK, Cohen EL, Messing R, Munro HN, Wurtman RJ. Relevance of free tryptophan in serum to tissue tryptophan concentrations. Metabolism: clinical and experimental. 1974; 23(12):1107-16.

10. Knott PJ, Curzon G. Free tryptophan in plasma and brain tryptophan metabolism. Nature, New Bio. 1972; 239, 452453.

11. Curzon G, Friedel J, Knott PJ. The effect of fatty acids on the binding of tryptophan to plasma protein. Nature. 1974; 242(5394):198-200.

12. Allegri G, Costa CV, Bertazzo A, Biasiolo M, Ragazzi E. Enzyme activities of tryptophan metabolism along the kynurenine pathway in various species of animals. Il Farmaco. 2003; 58(9):829-36.

13. Ruddick JP, Evans AK, Nutt DJ, Lightman SL, Rook GA, Lowry CA. Tryptophan metabolism in the central nervous system: medical implications. Expert reviews in molecular medicine. 2006; 8(20):1-27.

14. Botting NP. Chemistry and neurochemistry of the kynurenine pathway of tryptophan metabolism. Chemical Society Reviews. 1995; 24(6):401-12.

15. Leblhuber F, Geisler S, Steiner K, Fuchs D, Schutz B. Elevated fecal calprotectin in patients with Alzheimer's dementia indicates leaky gut. J Neural Transm (Vienna). 2015; 122 (9):1319-22.

16. Velloso NA, Dalmolin GD, Gomes GM, Rubin MA, Canas PM, Cunha RA, et al. Spermine improves recognition memory deficit in a rodent model of Huntington's disease. Neurobiology of learning and memory. 2009; 92(4):574-80.

17. Veres G, Molnar M, Zadori D, Szentirmai M, Szalardy L, Torok R, et al. Central nervous systemspecific alterations in the tryptophan metabolism in the 3-nitropropionic acid model of Huntington's disease. Pharmacology, biochemistry, and behavior. 2015; 132:115-24.

18. Favre D, Mold J, Hunt PW, Kanwar B, Loke P, Seu L, et al. Tryptophan catabolism by indoleamine 2,3-dioxygenase 1 alters the balance of TH17 to regulatory T cells in HIV disease. Science translational medicine. 2010; 2(32):32ra6.

19. Miller AH, Haroon E, Raison CL, Felger JC. Cytokine targets in the brain: impact on neurotransmitters and neurocircuits. Depression and anxiety. 2013; 30(4):297-306.

20. Veen C, Myint AM, Burgerhout KM, Schwarz MJ, Schutze G, Kushner SA, et al. Tryptophan pathway alterations in the postpartum period and in acute postpartum psychosis and depression. Journal of affective disorders. 2016; 189:298-305.

21. Li Y, Eskelund AR, Zhou H, Budac DP, Sanchez C, Gulinello M. Behavioral deficits are accompanied by immunological and neurochemical changes in a mouse model for neuropsychiatric lupus (NP-SLE). International journal of molecular sciences. 2015; 16(7):15150-71.

22. Snyder MS. Effects of Tryptophan metabolism on inflammation in human skin. Senior Projects Spring. 2015; 339. 
23. Wang Q, Liu D, Song P, Zou MH. Deregulated tryptophan-kynurenine pathway is linked to inflammation, oxidative stress, and immune activation pathway in cardiovascular diseases. Frontiers in bioscience (Landmark edition). 2015; 20:1116.

24. Müller N, Myint AM, Schwarz MJ. Kynurenine pathway in schizophrenia: pathophysiological and therapeutic aspects. Curr Pharm Des. 2011; 17(2):130-6. Review.

25. Reininghaus EZ, McIntyre RS, Reininghaus B, Geisler S, Bengesser SA, Lackner N, et al. Tryptophan breakdown is increased in euthymic overweight individuals with bipolar disorder: a preliminary report. Bipolar disorders. 2014; 16(4):432-40.

26. Stoll J, Goldman D. Isolation and Structural Characterization of the Murine Tryptophan Hydroxylase Gene. Laboratory of Clinical Studies, National Institute on alcohol abuse and alcoholism, Bethesda, MD. Journal of Neuroscience Research. 1991; 28: 457-465.

27. Steinhilber D. Melatonin, melatonin receptor agonists and tryptophan as sleep aids. Pharm Unserer Zeit. 2007; 36(3):213-7. Review.

28. Anderson G, Maes M. Melatonin: an overlooked factor in schizophrenia and in the inhibition of antipsychotic side effects. Metab Brain Dis. 2012; 27(2):113-9. Review.

29. Anderson G, Jacob A, Bellivier F, Geoffroy PA. Bipolar Disorder: The Role of the Kynurenine and Melatonergic Pathways. Curr Pharm Des. 2016; 22(8):987-1012. Review.

30. Azad MC, Shoesmith WD, Al Mamun M, Abdullah AF, Naing DK, Cardiovascular diseases among patients with schizophrenia. Phanindranath M, Turin TC. Asian J Psychiatr. 2016; 19:28-36. Review.

31. Yang Y, Sauve AA. NAD+ metabolism: Bioenergetics, signaling and manipulation for therapy. Biochimica et biophysica acta. 2016; 1864(12):1787-800.

32. Chiappelli J, Postolache TT, Kochunov P, Rowland LM, Wijtenburg SA, Shukla DK, et al. Tryptophan Metabolism and White Matter Integrity in Schizophrenia. Neuropsychopharmacology: official publication of the American College of Neuropsychopharmacology. 2016; 41(10):2587-95.

33. McCusker RH, Kavelaars A, Heijnen CJ, Dantzer R, Kelley KW. Depression, inflammation and tryptophan metabolism. The Wiley-Blackwell Handbook of Psychoneuroimmunology. 2014; 448-68.

34. Erhardt S, Schwieler L, Imbeault S, Engberg G. The kynurenine pathway in schizophrenia and bipolar disorder Neuropharmacology. 2017; 112(Pt B):297-306.

35. Kanchanatawan B, Sirivichayakul S, Thika S, Ruxrungtham K, Carvalho AF, Geffard M, Anderson G, Noto C, Ivanova R, Maes M. Physio-somatic symptoms in schizophrenia: association with depression, anxiety, neurocognitive deficits and the tryptophan catabolite pathway. Metab Brain Dis. 2017; 1-14.

36. Ball HJ, Jusof FF, Bakmiwewa SM, Hunt NH, Yuasa HJ. Tryptophan-catabolizing enzymes - party of three. Frontiers in immunology. 2014; $5: 485$.

37. Grant RS, Naif H, Thuruthyil SJ, Nasr N, Littlejohn T, Takikawa O, et al. Induction of indoleamine 2, 3-dioxygenase in primary human macrophages by HIV-1. Redox Report. 2013; 5:(2-3); 105-107.

38. Larkin PB, Sathyasaikumar KV, Notarangelo FM, Funakoshi H, Nakamura T, Schwarcz R, et al. Tryptophan 2, 3-dioxygenase and indoleamine 2, 3-dioxygenase 1 make separate, tissue-specific contributions to basal and inflammation-induced kynurenine pathway metabolism in mice. Biochimica et Biophysica Acta (BBA)-General Subjects. 2016; 1860(11):2345-54.

39. Moresco RM, Lavazza T, Belloli S, Lecchi M, Pezzola A, Todde S, et al. Quinolinic acid induced neurodegeneration in the striatum: a combined in vivo and in vitro analysis of receptor changes and microglia activation. European journal of nuclear medicine and molecular imaging. 2008; 35(4):704-15.

40. Iaccarino HF, Suckow RF, Xie S, Bucci DJ. The effect of transient increases in kynurenic acid and quinolinic acid levels early in life on behavior in adulthood: Implications for schizophrenia. Schizophr Res. 2013; 150(2-3):392-7.

41. Pocivavsek A, Wu HQ, Elmer GI, Bruno JP, Schwarcz R. Pre-and postnatal exposure to kynurenine causes cognitive deficits in adulthood. Eur J Neurosci. 2012; 35(10):1605-12.

42. Owe-Young R, Webster NL, Mukhtar M, Pomerantz RJ, Smythe G, Walker D, et al. Kynurenine pathway metabolism in human blood-brain-barrier cells: implications for immune tolerance and neurotoxicity. Journal of neurochemistry. 2008; 105(4):1346-57.

43. Speciale C, Hares K, Schwarcz R, Brookes N. High-affinity uptake of L-kynurenine by a $\mathrm{Na}^{+}-$ independent transporter of neutral amino acids in astrocytes. The Journal of neuroscience: the official journal of the Society for Neuroscience. 1989; 9(6): 2066-72. 
44. Perez-De La Cruz V, Carrillo-Mora P, Santamaria A. Quinolinic Acid, an endogenous molecule combining excitotoxicity, oxidative stress and other toxic mechanisms. International journal of tryptophan research: IJTR. 2012; 5:1-8.

45. Condray R, Dougherty GG Jr, Keshavan MS, Reddy RD, Haas GL, Montrose DM, et al. 3Hydroxykynurenine and clinical symptoms in first-episode neuroleptic-naive patients with schizophrenia. Int J Neuropsychopharmacol. 2011; 14(6):756-67.

46. Okuda S, Nishiyama N, Saito H, Katsuki H. 3-Hydroxykynurenine, an endogenous oxidative stress generator, causes neuronal cell death with apoptotic features and region selectivity. Journal of neurochemistry. 1998; 70(1):299-307.

47. Wichers MC, Maes M. The role of indoleamine 2,3-dioxygenase (IDO) in the pathophysiology of interferon-alpha-induced depression. Journal of psychiatry \& neuroscience: JPN. 2004; 29(1):11-7.

48. Stone TW, Stoy N, Darlington LG. An expanding range of targets for kynurenine metabolites of tryptophan. Trends in pharmacological sciences. 2013; 34(2):136

49. Carvajal FJ, Mattison HA, Cerpa W. Role of NMDA Receptor-Mediated Glutamatergic Signaling in Chronic and Acute Neuropathologies. Neural plasticity. 2016; 2016:2701526.

50. Petrovchich I, Sosinsky A, Konde A, Archibald A, Henderson D, Maletic-Savatic M, et al. Metabolomics in Schizophrenia and Major Depressive Disorder. Frontiers in Biology. 2016; 11(3):22231.

51. Carpenedo R, Pittaluga A, Cozzi A, Attucci S, Galli A, Raiteri M, et al. Presynaptic kynurenatesensitive receptors inhibit glutamate release. The European journal of neuroscience. 2001; 13(11):2141-7.

52. Rassoulpour A, Wu HQ, Ferre S, Schwarcz R. Nanomolar concentrations of kynurenic acid reduce extracellular dopamine levels in the striatum. Journal of neurochemistry. 2005; 93(3):762-5.

53. Ohshiro H, Tonai-Kachi H, Ichikawa K. GPR35 is a functional receptor in rat dorsal root ganglion neurons. Biochemical and biophysical research communications. 2008; 365(2):344-8.

54. Goldstein LE, Leopold MC, Huang X, Atwood CS, Saunders AJ, Hartshorn M, et al. 3Hydroxykynurenine and 3-hydroxyanthranilic acid generate hydrogen peroxide and promote alphacrystallin cross-linking by metal ion reduction. Biochemistry. 2000; 39(24):7266-75.

55. Bitzer-Quintero OK, Davalos-Marin AJ, Ortiz GG, Meza AR, Torres-Mendoza BM, Robles RG, et al. Antioxidant activity of tryptophan in rats under experimental endotoxic shock. Biomedicine \& pharmacotherapy = Biomedecine \& pharmacotherapie. 2010; 64(1):77-81.

56. Kroenke K, Wu J, Bair MJ, Krebs EE, Damush TM, Tu W. Reciprocal relationship between pain and depression: a 12-month longitudinal analysis in primary care. The Journal of Pain. 2011; 12(9):964-73.

57. Felger, JC \& Treadway, MT. Inflammation effects on motivation and motor activity: role of dopamine. Neuropsychopharmacology. 2017; 42(1), 216-241.

58. Erhardt, S., Schwieler, L., Imbeault, S., \& Engberg, G.The kynurenine pathway in schizophrenia and bipolar disorder. Neuropharmacology. 2017; 112: 297-306.

59. Weinberger DR. Implications of normal brain development for the pathogenesis of schizophrenia. Arch Gen Psychiatry 1987; 44(7): 660-9.

60. Barry S, Clarke G, Scully P, Dinan TG. Kynurenine pathway in psychosis: evidence of increased tryptophan degradation. J Psychopharmacol. 2009; 23(3):287-94.

61. Skogh E, Lundberg K. Increased levels of IL-6 in the cerebrospinal fluid of patients with chronic schizophrenia-significance for activation of the kynurenine pathway. Journal of psychiatry \& neuroscience: JPN. 2015; 40(2):126.

62. Lee M, Jayathilake K, Dai J, Meltzer HY. Decreased plasma tryptophan and tryptophan/large neutral amino acid ratio in patients with neuroleptic-resistant schizophrenia: relationship to plasma cortisol concentration. Psychiatry Res. 2011; 185(3):328-33.

63. Sathyasaikumar KV, Stachowski EK, Wonodi I, Roberts RC, Rassoulpour A, McMahon RP, et al. Impaired kynurenine pathway metabolism in the prefrontal cortex of individuals with schizophrenia. Schizophrenia bulletin. 2011; 37(6):1147-56.

64. Ravikumar A, Deepadevi KV, Arun P, Manojkumar V, Kurup PA, Tryptophan. and tyrosine catabolic pattern in neuropsychiatric disorders. Neurol. India. 2000; 48 (3), 231-238.

65. Myint AM, Kim YK, Verkerk R, Park SH, Scharpé S, Steinbusch HW, Leonard BE. Tryptophan breakdown pathway in bipolar mania. J Affect Disord. 2007; 102(1-3):65-72. 
66. Myint AM, Schwarz MJ, Verkerk R, Mueller HH, Zach J, Scharpé S, Steinbusch HW, Leonard BE, Kim YK. Reversal of imbalance between kynurenic acid and 3 hydroxykynurenine by antipsychotics in medication-naïve and medication-free schizophrenic patients. Brain Behav Immun. 2011; 25(8):1576-81.

67. Fukui S, Schwarcz R, Rapoport SI, Takada Y, Smith QR. Blood-brain barrier transport of kynurenines: implications for brain synthesis and metabolism. J Neurochem. 1991; 56(6):2007-2017.

68. Oxenkrug G, van der Hart M, Roeser J, Summergrad P. Anthranilic Acid: A New Biomarker and Treatment Target for Schizophrenia. Ann Psychiatry Ment Health. 2016; 4(2):1059- 1064.

69. Condray R, Dougherty Jr GG, Keshavan MS, Reddy RD, Haas GL, Montrose DM, et al. 3Hydroxykynurenine and clinical symptoms in first-episode neuroleptic-naive patients with schizophrenia. The International Journal of Neuropsychopharmacology. 2011; 14(6):756-67. 